\title{
Light hadron spectrum and quark masses in $2+1$ flavor QCD
}

\section{CP-PACS and JLQCD Collaborations:}

T. Ishikawa ${ }^{a * \dagger}$, S. Aoki ${ }^{b, c}$, O. Bär ${ }^{b}$, M. Fukugita ${ }^{d}$, S. Hashimoto ${ }^{e, f}$, K.-I. Ishikawa ${ }^{g}$, N. Ishizuka ${ }^{a, b}$, Y. Iwasaki ${ }^{b}$, K. Kanaya ${ }^{b}$, T. Kaneko ${ }^{e, f}$, Y. Kuramashi ${ }^{a, b}$, M. Okawa ${ }^{g}$, Y. Taniguchi ${ }^{a, b}$, N. Tsutsui ${ }^{e}$, A. Ukawa ${ }^{a, b}$ and T. Yoshié To $^{a, b}$

${ }^{a}$ Center for Computational Sciences, University of Tsukuba, Tsukuba, Ibaraki 305-8577, Japan

${ }^{b}$ Graduate School of Pure and Applied Sciences, University of Tsukuba, Tsukuba, Ibaraki 305-8571, Japan

${ }^{c}$ Riken BNL Research Center, Brookhaven National Laboratory, Upton, New York 11973, USA

${ }^{d}$ Institute for Cosmic Ray Research, University of Tokyo, Kashiwa 277-8582, Japan

${ }^{e}$ High Energy Accelerator Research Organization (KEK), Tsukuba 305-0801, Japan

${ }^{f}$ School of High Energy Accelerator Science, The Graduate University for Advanced Studies

(Sokendai), Tsukuba 305-0801, Japan

${ }^{g}$ Department of Physics, Hiroshima University, Higashi-Hiroshima, Hiroshima 739-8526, Japan

CP-PACS and JLQCD collaborations are carrying out a joint project of the $2+1$ flavor full QCD simulation. Gauge configurations are generated for the non-perturbatively $O(a)$-improved Wilson quark action and the Iwasaki gauge action using PHMC algorithm at three lattice spacings, $a \sim$ $0.076,0.010$ and $0.122 \mathrm{fm}$, with a fixed physical volume $(2.0 \mathrm{fm})^{3}$. We present analysis for the light meson spectrum and quark masses in the continuum limit, which are determined using data obtained from the simulations at the two coarser lattices. Our simulations reproduce experimental values of meson masses. The ud and strange quark masses turn out to be $m_{u d}^{\overline{M S}}(\mu=2 \mathrm{GeV})=$ 3.34(23) $\mathrm{MeV}$ and $m_{s}^{\overline{M S}}(\mu=2 \mathrm{GeV})=86.7(5.9) \mathrm{MeV}$. We also show preliminary results at our finest lattice spacing for which simulations are still being continued.

XXIIIrd International Symposium on Lattice Field Theory

25-30 July 2005

Trinity College, Dublin, Ireland

\footnotetext{
* Speaker.

†E-mail: tomomi@ccs.tsukuba.ac.jp
} 


\begin{tabular}{ccccc}
\hline$\beta$ & size & $a[\mathrm{fm}](K$-input $)$ & $a[\mathrm{fm}](\phi$-input $)$ & trajectory \\
\hline 1.83 & $16^{3} \times 32$ & $0.1222(17)$ & $0.1233(30)$ & $7000-8600$ \\
1.90 & $20^{3} \times 40$ & $0.0993(19)$ & $0.0995(19)$ & $5000-9200$ \\
2.05 & $28^{3} \times 56$ & $0.0758(48)$ & $0.0755(48)$ & $3000-4000$ \\
\hline
\end{tabular}

Table 1: Simulation parameters. The production run at $\beta=2.05$ is still in progress.

\section{Introduction}

The calculation of the light hadron spectrum and quark masses is the most fundamental issue in lattice QCD simulations. So far, systematic studies made in quenched [1] and two flavor $\left(N_{f}=2\right)$ full QCD [2] revealed that 1) the $O(10 \%)$ deviation of the quenched spectrum from experiment is largely reduced in $N_{f}=2 \mathrm{QCD}$, and 2) dynamical up and down quarks reduce significantly the quark masses. As a next step in this direction, the CP-PACS and JLQCD collaborations are carrying out a $2+1$ flavor $\left(N_{f}=2+1\right)$ full QCD simulation project [3], in which degenerate up and down ("light") quarks and a strange quark are treated dynamically. With this simulation, we hope to obtain the spectrum and quark masses much closer to those of the QCD with no approximation.

In this article, we present the results in the continuum limit extrapolated from two lattice spacings together with preliminary results obtained by on-going simulations at our finest lattice spacing. In this study, we employ the Wilson quark formalism as in our previous works, because this formalism has no ambiguity in quark-flavor interpretation. We note that a similar attempt is being made [4] with the staggered quark formalism.

\section{Gauge configuration generation}

For the lattice action, we employ the renormalization group (RG) improved Iwasaki gauge action and the clover quark action with the improvement coefficient $c_{S W}$ determined non-perturbatively for the RG action [5]. The choice of the gauge action is made to avoid a first-order phase transition (lattice artifact) observed for the plaquette gauge action [6].

Configurations are generated with the Polynomial Hybrid Monte Carlo (PHMC) algorithm. (See Ref.[7] for our implementation.) The time step $\delta \tau$ in the molecular dynamics and the order $N_{\text {poly }}$ of Chebyshev polynomial used for an approximation of quark determinant are chosen such that the HMC and the global Metropolis acceptance rate achieves $85 \%$ and $90 \%$, respectively.

Simulations are performed at three values of the coupling constant chosen so that the square of the lattice spacing $a^{2}$ is placed at an even interval. The physical volume is fixed at $(2.0 \mathrm{fm})^{3}$. Simulation parameters are listed in Table 1. At each coupling, we generate configurations for ten combinations of hopping parameters $\left(\kappa_{u d}, \kappa_{s}\right)$, five for the ud quark mass taken in the range $m_{P S} / m_{V} \sim 0.6-0.78$ and two for the strange quark mass chosen around $m_{P S} / m_{V} \sim 0.7$. Gauge configuration generation has already been finished at the two coarser lattices. We plan to continue the on-going simulation at the finest lattice up to 6000 trajectories. 


\section{Measurement and analysis}

Measurements are performed at every 10 trajectories. We first fix the configuration to the Coulomb gauge and then calculate quark propagators for valence quark masses taken equal to one of sea quark masses, using point and exponentially smeared sources and sinks. For analysis, we use the combination of smeared source and point sink, because with this combination effective masses reach a plateau earliest and the statistical error is the smallest. Meson masses and quark masses are determined from single mass $\chi^{2}$ fits to correlators $\langle P(t) P(0)\rangle,\langle V(t) V(0)\rangle$ and $\left\langle A_{4}(t) P(0)\right\rangle$, where $P, V$ and $A_{\mu}$ denote the pseudoscalar, the vector and the one-loop $O(a)$-improved axial-vector current, respectively. We include correlations in time but ignore correlation among correlators. Errors are estimated by the binned jackknife method with a bin size of 100 trajectories.

Chiral fits are made to light-light, light-strange and strange-strange meson masses simultaneously ignoring their correlations, using a quadratic polynomial function in terms of the sea and valence quark masses. ${ }^{1}$ For chiral fits, we use two definitions of the quark mass. One is the vector Ward identity (VWI) quark mass defined by $m_{q}^{V W I}=\left(1 / \kappa-1 / \kappa_{c}\right) / 2$, where $\kappa_{c}$ is the critical hopping parameter at which $m_{P S}$ at $\kappa_{u d}=\kappa_{s}=\kappa_{v a l}=\kappa_{c}$ vanishes. The other is the axial-vector Ward identity (AWI) quark mass defined by $m_{q}^{A W I}=\nabla_{\mu} A_{\mu}(x) /(2 P(x))$. The lattice spacing, the meson masses and $m_{q}^{V W I}$ at the physical point are determined from the chiral fit in terms of $m_{q}^{V W I}$, while $m_{q}^{A W I}$ at the physical point are from the chiral fit in terms of $m_{q}^{A W I}$. The lattice spacing and the meson masses obtained from the latter chiral fit are consistent with those from the former.

For the physical point, we consider two cases. One is called as " $K$-input" in which we take the experimental values $m_{\pi}=0.1350 \mathrm{GeV}, m_{\rho}=0.7684 \mathrm{GeV}$ and $m_{K}=0.4977 \mathrm{GeV}$ as inputs. The other is " $\phi$-input" in which $m_{\pi}, m_{\rho}$ and $m_{\phi}=1.0194 \mathrm{GeV}$ are taken as inputs. The lattice spacings determined from the $K$ - and $\phi$ - inputs are consistent with each other, as shown in Table 1 .

\section{Light meson spectrum}

We evaluate the meson masses in the continuum limit extrapolating the data linearly in $a^{2}$ at two coarser lattices, for which runs and measurements are completed. The results shown in Fig. 1 are consistent with experiment though errors are still large. Taking $K^{*}$ with $K$-input as an example, the linear fit is given by $m_{K^{*}}=m_{0}\left(1+c\left(\Lambda_{Q C D} \cdot a\right)^{2}\right)$ with $m_{0}=897(10) \mathrm{MeV}$ and $c=-1.38(78)$ where we assumed $\Lambda_{Q C D}=200 \mathrm{MeV}$. The $\mathrm{O}(1)$ magnitude of the coefficient $c$ is reasonable, albeit apparently larger scaling violation is somewhat disappointing, in comparison to quenched(triangles) and $N_{f}=2$ (squares) data [2] which are based on only the tadpole-improved action. The points at the finest lattice spacing are based on measurements for 3000 trajectories. Statistics are still low. We hope we continue simulations up to the full 6000 trajectories and clarify the continuum extrapolation in the near future.

\section{Light quark masses}

The physical quark mass is determined for the $\overline{\mathrm{MS}}$ scheme at the scale $\mu=2 \mathrm{GeV}$. To do

\footnotetext{
${ }^{1}$ Chiral fit is one of the important sources of systematic errors. Recently, Wilson chiral perturbation theory (WChPT) has been proposed, in which infrared chiral logarithms and finite lattice spacing corrections to them for the Wilson quark action are incorporated. An application of WChPT to our $N_{f}=2+1$ QCD data is in progress. (See Ref. [8].)
} 

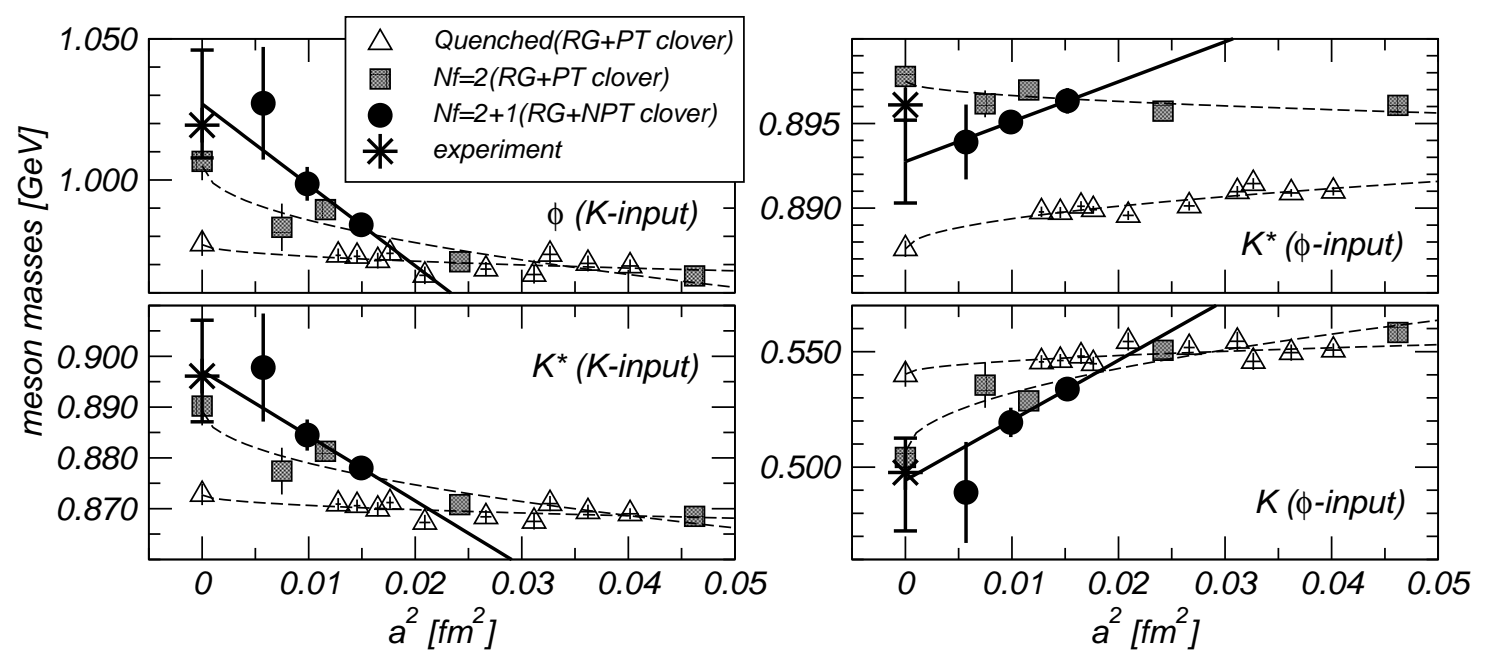

Figure 1: Continuum extrapolation of meson masses, compared with those for quenched and $N_{f}=2$ QCD [2]. Note that the quenched and $N_{f}=2$ simulations are made with the one-loop perturbatively $O(a)$ improved clover action. Thus extrapolations are made linearly in $a$.

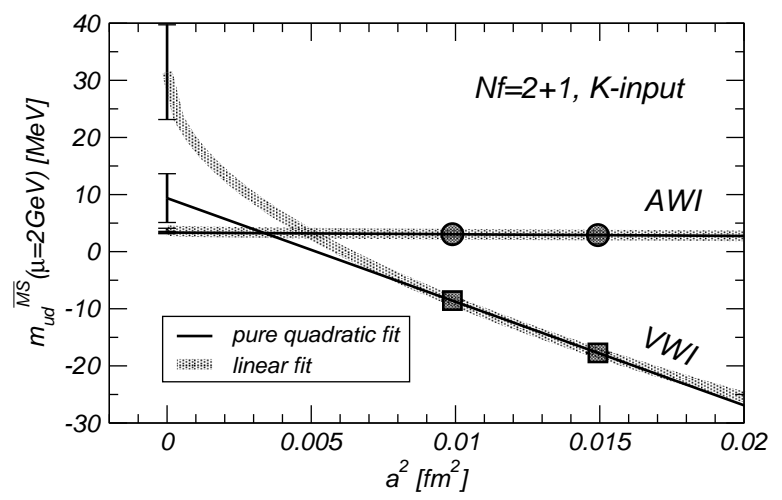

Figure 2: Continuum extrapolations of the up and down quark mass defined by the AWI and VWI ( $K$-input).

this, we first translate the quark mass to that in the $\overline{\mathrm{MS}}$ scheme at scale $\mu=a^{-1}$ using $Z$-factors determined by tadpole-improved one-loop perturbation theory [9]. The renormalized quark masses are then evolved to $\mu=2 \mathrm{GeV}$ using the four-loop RG-equation.

In principle, the quark mass calculated in our way can have $O\left(g^{4} a\right)$ scaling violation since we use the matching factor and the improvement coefficient $c_{A}$ of the axial-vector current determined by one-loop perturbation theory. We compare in Fig. 2 the ud quark masses calculated with the VWI and AWI definitions. They do not agree at all in the continuum limit, when they are extrapolated in $a$. On the other hand, pure quadratic fits yield reasonably consistent values. This suggests that terms linear in $a$ are small for ud quark masses. Similarly, the pure quadratic extrapolations of the VWI and AWI strange quark masses give a common value in the continuum limit.

We take the AWI definition to quote the continuum limit for estimates of the quark mass, since the magnitude of scaling violation is smaller for $m_{q}^{A W I}$ than for $m_{q}^{V W I}$, and thus the error in the 

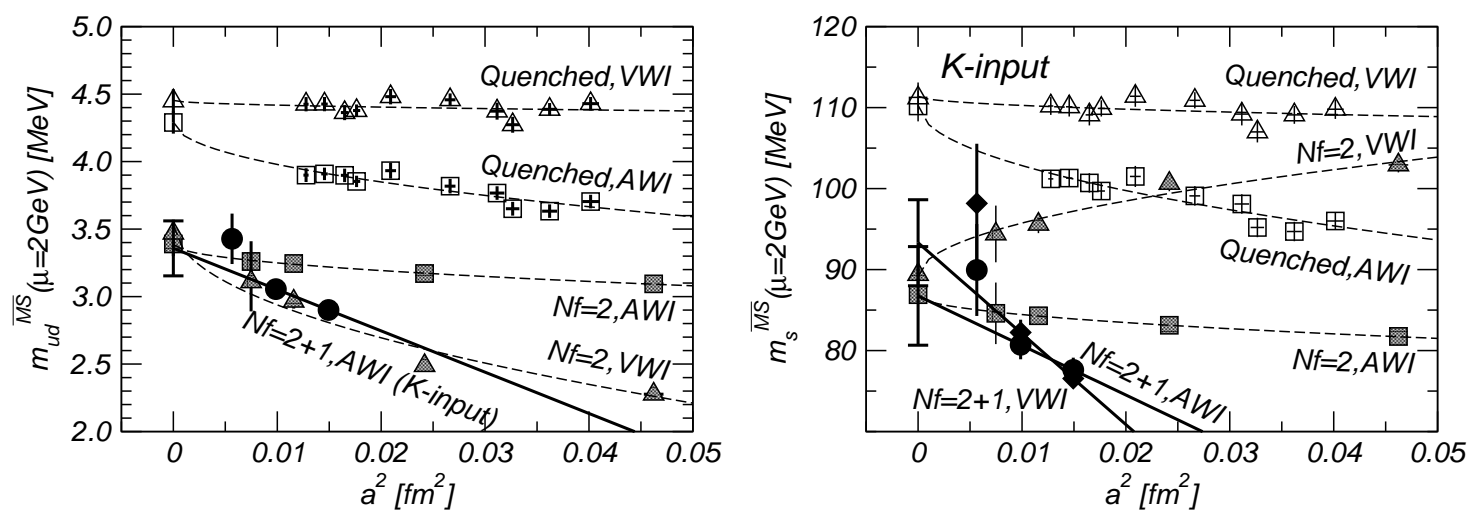

Figure 3: Continuum extrapolations of the up, down and strange quark masses obtained with the $K$-input. The data at the finest lattice is not included in the continuum extrapolations. For comparison, results for quenched and $N_{f}=2$ QCD are overlaid.

continuum limit is smaller for $m_{q}^{A W I}$, as shown in Fig. $2 .^{2}$ Note that this property is also observed in $N_{f}=2$ QCD.

In Fig. 3, we present continuum extrapolations of the up, down and strange quark masses determined with the $K$-input. The ud quark mass with the $\phi$-input agrees with the $K$-input at each lattice spacing. For the strange quark mass, the $K$ - and the $\phi$-inputs give different values at finite lattice spacings, but masses are extrapolated to a common value in the continuum limit, as observed in $N_{f}=2$ QCD. Thus we estimate the quark masses in the continuum limit from a combined fit to data with the $K$ - and the $\phi$-inputs,

$$
m_{u d}^{\overline{M S}}(\mu=2 \mathrm{GeV})=3.34(23) \mathrm{MeV}, \quad m_{s}^{\overline{M S}}(\mu=2 \mathrm{GeV})=86.7(5.9) \mathrm{MeV}
$$

In the continuum limit, the quark masses estimated for the $N_{f}=2+1$ QCD do not show deviations beyond statistical errors from those of $N_{f}=2 \mathrm{QCD}$.

\section{Conclusions and future plans}

In this article we have reported the status of our Wilson-clover $N_{f}=2+1$ simulations. The spectrum at two lattice spacings, when extrapolated to the continuum limit are consistent with experiment. However, the outcome of the on-going runs at the finest lattice spacing at $a \sim 0.076 \mathrm{fm}$, which we hope to complete in a half a year or so, is needed for a precise conclusion. The same comment also applies to light quark masses. A shortcoming of the current runs is a relatively large value of the dynamical up and down quark masses. We hope to bring improvement in our program by a combined application of the improved algorithm provided by the domain decomposition idea [10] and an enhanced computing power to be provided by PACS-CS, the successor to the CP-PACS computer.

\footnotetext{
${ }^{2}$ The VWI quark mass for the ud quarks is negative at our simulation points. This originates from a lack of chiral symmetry of the Wilson quark action. This is another reason to prefer the AWI definition.
} 


\section{Acknowledgments}

This work is supported by the Epoch Making Simulation Projects of Earth Simulator Center, the Large Scale Simulation Program No. 132 (FY2005) of High Energy Accelerator Research Organization (KEK), the Large Scale Simulation Projects of Academic Computing and Communications Center, University of Tsukuba, Super Sinet Projects of National Institute of Infomatics, and also by the Grant-in-Aid of the Ministry of Education (Nos. 13135204, 13640260, 14046202, 14740173, 15204015, 15540251, 15540279, 15740134, 16028201, 16540228, 17340066).

\section{References}

[1] S. Aoki et al. [CP-PACS Collaboration], Quenched Light Hadron Spectrum, Phys. Rev. Lett. 84 (2000) 238, [hep-lat/9904012]; Light hadron spectrum and quark masses from quenched lattice QCD, Phys. Rev. D67 (2003) 034503, [hep-lat/ 0206009 ].

[2] A. Ali Khan et al. [CP-PACS Collaboration], Dynamical Quark Effects on Light Quark Masses, Phys. Rev. Lett. 85 (2000) 4674, Phys. Rev. Lett. 90 (2003) 029902(E), [hep-lat / 000401 0]; Light hadron spectroscopy with two flavors of dynamical quarks on the lattice, Phys. Rev. D65 (2002) 054505, Phys. Rev. D67 (2003) 059901(E), [hep-lat/ 0105015 ].

[3] T. Kaneko et al. [CP-PACS and JLQCD Collaborations], Light hadron spectrum in three-flavor QCD with O(a)-improved Wilson quark action, Nucl. Phys. B (Proc. Suppl.) 129 (2004) 188, [hep-lat/0309137]; T. Ishikawa et al. [CP-PACS and JLQCD Collaborations], Light hadron spectrum in 2+1 flavor full QCD by CP-PACS and JLQCD Collaborations, Nucl. Phys. B (Proc. Suppl.) 140 (2005) 225, [hep-lat / 0409124$].$

[4] C. Aubin et al. [HPQCD Collaboration, MILC Collaboration and UKQCD Collaboration], First determination of the strange and light quark masses from full lattice QCD, Phys. Rev. D70 (2004) 031504(R), [hep-lat/ 0405022 ]; Light pseudoscalar decay constants, quark masses, and low energy constants from three-flavor lattice QCD, Phys. Rev. D70 (2004) 114501, [hep-lat/0407028].

[5] S. Aoki et al. [CP-PACS and JLQCD Collaborations], Nonperturbative $O($ a) improvement of the Wilson quark action with the RG-improved gauge action using the Schrödinger functional method, [hep-lat/0508031].

[6] S. Aoki et al. [JLQCD Collaboration], Bulk first-order phase transition in three-flavor lattice QCD with $O($ a)-improved Wilson fermion action at zero temperature, To be published in Phys. Rev. D, [hep-lat/0409016].

[7] S. Aoki et al. [JLQCD Collaboration], Polynomial hybrid Monte Carlo algorithm for lattice QCD with an odd number of flavors, Phys. Rev. D65 (2002) 094507, [hep-lat/0112051].

[8] S. Aoki, Chiral perturbation theory with Wilson-type fermions including a effects: $N_{f}=2$ degenerate case, Phys. Rev. D68 (2003) 054508, [hep-lat/0306027]; S. Aoki et al., Pseudo scalar meson masses in Wilson Chiral Perturbation Theory for 2+1 flavors, [hep-1at/ 0509049 ].

[9] S. Aoki et al., Perturbative renormalization factors of bilinear quark operators for improved gluon and quark actions in lattice QCD, Phys. Rev. $D 58$ (1998) 074505, [hep-lat/9802034].

[10] M. Lüscher, Lattice QCD and the Schwarz alternating procedure, JHEP 05 (2003) 052 , [hep-lat/ 0304007$]$; Solution of the Dirac equation in lattice QCD using a domain decomposition method, Comput. Phys. Commun. 156 (2004) 209, [hep-lat/ 0310048 ]; Schwarz-preconditioned HMC algorithm for two-flavour lattice QCD, [hep-lat/ 0409106 ]. 